\title{
The magnificent night sky - why it must be protected from light pollution
}

\author{
Richard J. Wainscoat \\ University of Hawaii, Institute for Astronomy \\ 2680 Woodlawn Drive, Honolulu, HI 96822, USA \\ email: rjw@ifa.hawaii.edu
}

\begin{abstract}
The view of the night sky from most of the populated regions of Earth is now seriously compromised by light pollution. An entire generation of citizens is now growing up without ever having the opportunity to gaze upon the magnificent night sky from a dark location. Much of modern astronomy involves study of faint objects, often with brightnesses similar to or fainter than the night sky. Astronomers are therefore extremely vulnerable to increases in sky brightness from light pollution. Despite their remote locations, the dark night skies over the major optical observatories are increasingly threatened by growth of artificial light at night.
\end{abstract}

Keywords. light pollution, telescopes, observatories, sky brightness

\section{Introduction}

Modern society has come to expect lighting at night. Many inefficient light fixtures direct some of their light directly into the sky. Light is also reflected from the ground into the sky. Scattering from air molecules (Rayleigh scattering) and from aerosols (Mie scattering) redirects some of this light back downwards, producing an artificial brightening of the night sky (sky glow). Astronomers are severely affected by light pollution. Every $10 \%$ brighter the night sky becomes requires astronomers to spend $10 \%$ more time making their observations (i.e., it makes their telescopes' effective apertures $10 \%$ smaller). Increased sky brightness will render the faintest sources unobservable because of practical time constraints.

Light pollution describes the adverse effects of man-made lighting. It includes sky glow, glare, energy waste, light trespass, decreased visibility at night, and environmental harm. The aspect of light pollution that most affects astronomers is the increase in sky glow, which makes it harder to see faint objects.

Light in the dark night sky has approximately equal contributions from

- Starlight;

- Zodiacal light - sunlight scattered from dust in the solar system (dust from comets and asteroid collisions); and

- Airglow - high-altitude atmospheric emissions caused by solar radiation. The brightness of the airglow varies with solar activity; it is brighter near solar maximum, and fainter near solar minimum.

Artificial light quickly overwhelms this delicate balance, and makes stars invisible. Figure 1 shows the natural night sky seen from Mauna Kea on a night with very little light pollution. The zodiacal light band is clearly visible.

The growth in lighting across the world is incessant, and is outpacing the growth in population. On the timescale of a year or so, it is hard to recognize, and is accepted by 


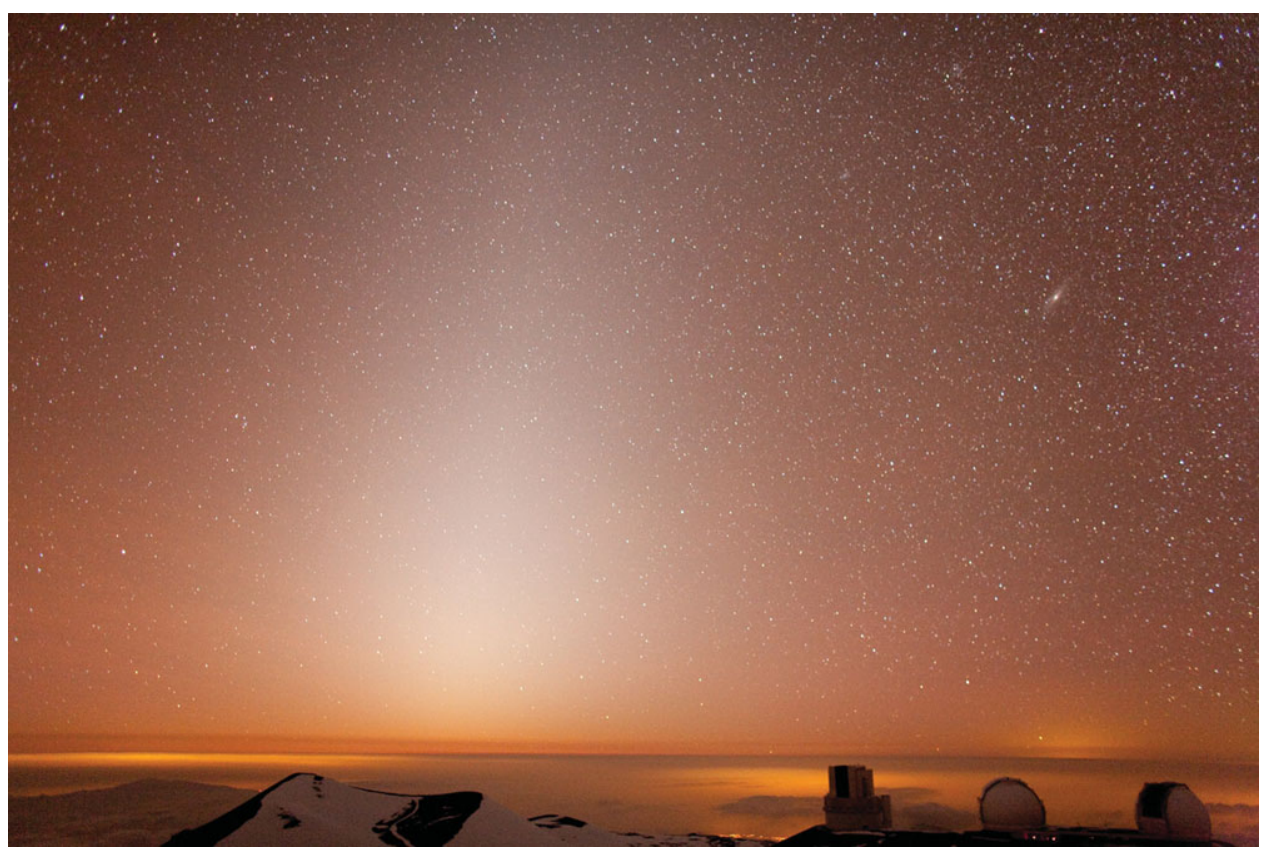

Figure 1. Zodiacal light band along the ecliptic seen from Mauna Kea. This photo was taken on a night when low clouds blanketed nearly all artificial lights across most of Hawaii. The glow from $300 \mathrm{~km}$ distant Honolulu is seen near the horizon on the right. Emission from zodiacal light is present across the whole sky; it is brighter along the ecliptic and at smaller solar elongations.

society. But on longer timescales, it is devastating. Some of the most important discoveries of modern astronomy came from Mt. Wilson Observatory, outside Los Angeles, in the early part of the 20th century. Light pollution from Los Angeles and the surrounding area has since rendered Mt. Wilson largely unusable for optical astronomy, and has seriously degraded the capability of more distant Palomar Observatory.

Many of our children are growing up without ever seeing a dark sky. In a dark sky, it is usually possible to see about 2,000 stars with the naked eye. Much of our population lives in urban areas, with perhaps no more than 200 stars visible at the edges of an urban area, and 20 or fewer stars visible nearer the center. You need to travel $100 \mathrm{~km}$ or more away from a large town or city to escape most of its glow; when urban settlements are spaced closer than this, the entire region becomes affected by light pollution. There are no areas in Europe that are unaffected by light pollution, and very few places east of the Mississippi in the United States. Figure 2 shows the Milky Way center seen from the dark sky over Mauna Kea. Many children have never seen the Milky Way. Inability to see the night sky may lead to lack of public interest in astronomy, and consequent reductions in public funding for astronomical research. The sight of the magnificent night sky has inspired some of our brightest minds to pursue careers in science.

\section{Lighting and society}

Lighting is commonly associated with safety in modern society. It is commonly regarded as a crime deterrent, and is increasingly being used as a form of advertising. Too often, the appearance of a light fixture in the daytime is more important than how well it works at night. Many cities use old-fashioned light fixtures that have been chosen to create a historical appearance, but they are very inefficient and create a lot of light pollution. 


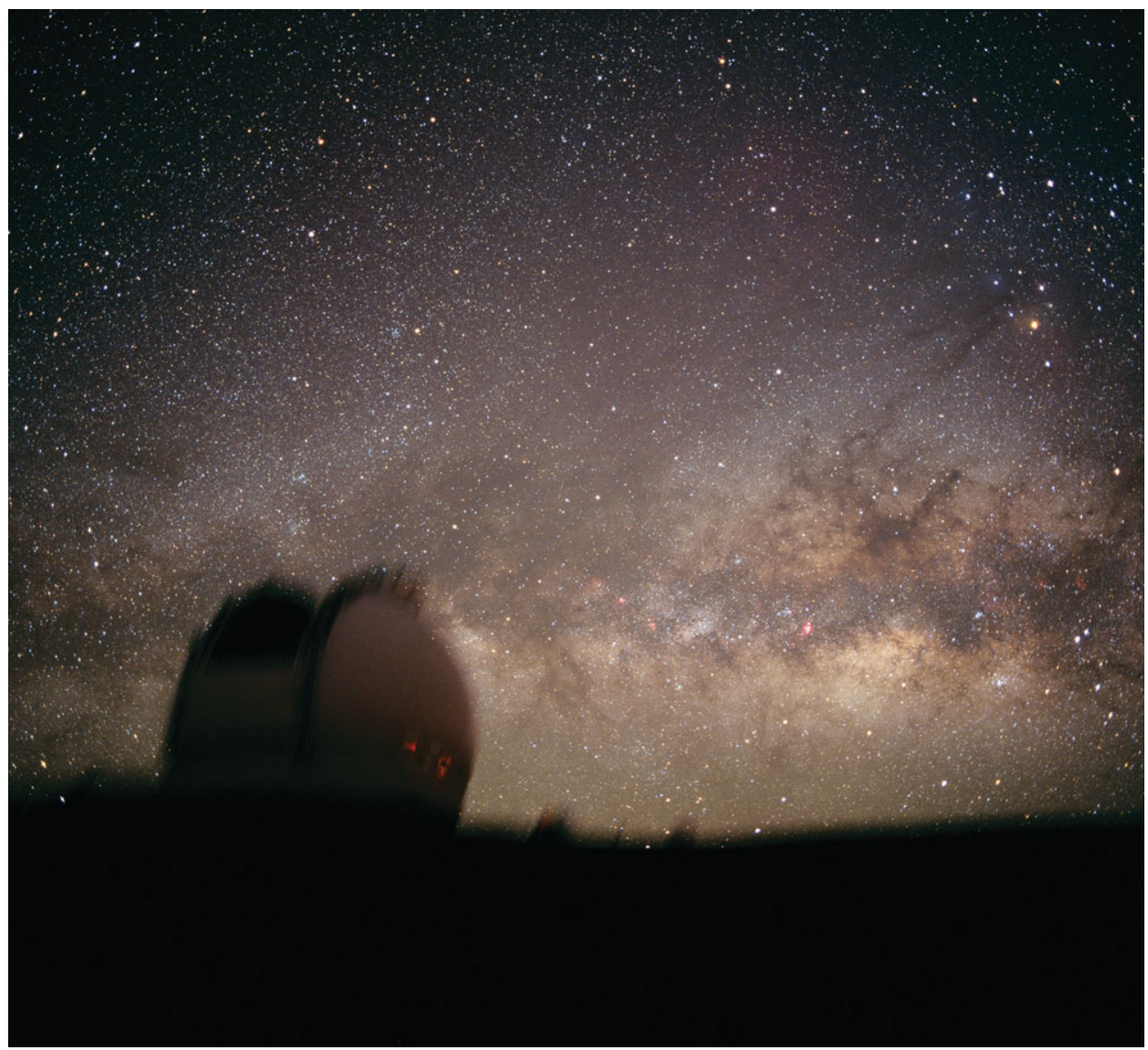

Figure 2. The Milky Way and the center of our Galaxy seen from Mauna Kea, Hawaii. The silhouette of the Keck I telescope dome is seen on the left. An increasing fraction of our population has never seen the Milky Way.

Fortunately, it is not just astronomers that are concerned about the growth of light pollution and the growing inability to see the night sky. The International Dark Sky Association has been active for many years in promoting good lighting practices. The ecological consequences of artificial light at night (Rich and Longcore 2006) are many and serious. Light controls the human circadian rhythm, and new links between excessive light at night and human health are broadening the interest in controlling light pollution.

Enormous amounts of energy are being wasted by inefficient and excessive use of light at night. Across the United States, more than $\$ 2$ billion in energy is wasted each year by poor lighting. Increasing public awareness of the need to conserve energy provides us with further leverage to reduce light pollution.

\section{The world's great observatory sites}

There is a common public misconception that astronomers' problems with light pollution can be solved from space. Although the sky seen from orbit is darker (the dominant background light source is zodiacal light), the cost of astronomy from space is enormous, and only small telescopes have ever been launched. The Hubble Space Telescope (HST) has an aperture of 2.4 meters, compared to the Keck telescopes' 10 meter aperture, with 
about 17 times as much collecting area. The proposed thirty-meter telescope will have an aperture 9 times larger still, and a resolving power with adaptive optics that far exceeds the capabilities of the HST. Instruments on ground-based telescopes are easily upgraded and repaired; it is difficult or impossible to repair telescopes in space. The vast majority of modern optical astronomy continues to be done from the ground.

There are only a limited number of really good places on Earth to do optical and infrared astronomy. Because of the prevailing direction of the circulation of Earth's atmosphere, the best places to do astronomy are typically on the west coasts of continents, or on isolated islands. These locations have less atmospheric turbulence, and therefore sharper images can be obtained. Because sources are faint, sharpness of images is critical to most modern astronomy.

Sites for astronomy need good weather, and therefore should not be located near the equator, or in the temperate wet regions. Telescopes should also be located on mountains, above the moist marine layer of air. The mountains on which they are located should be geologically stable (not active volcanos). The resultant list of good sites for astronomy is therefore:

- coastal mountains of northern Chile

- Mauna Kea and Haleakala in Hawaii

- La Palma in the Canary Islands

- coastal mountains of North America, including California, Baja California (Mexico), and extending east to Arizona

- one mountain in South Africa, and the plateau in Namibia

The early astronomy sites in California-Mt. Wilson, Lick, and Palomar are all excellent sites, but each now is seriously compromised by light pollution. The plateau in Namibia has not been evaluated for astronomy; it is not clear whether a plateau can deliver as good image quality as a mountain.

Beyond this list, it is clear that Antarctica, with its high altitude, dry air, and cold temperatures has special properties for astronomy (but support of astronomy endeavors in Antarctica is very costly, and only small telescopes are in use there). There are also some remote high sites in central Asia that may be good for astronomy, but have not yet been fully evaluated.

Since the entire sky cannot be seen from any one site, it is absolutely essential to preserve good sites in each hemisphere.

\section{Types of lighting}

Astronomers have for many years benefited from the energy efficiency of sodium lighting. Low-pressure sodium lights, still the most energy efficient light source, emit most of their light in the sodium doublet near $589 \mathrm{~nm}$. These are the best lights for use near observatories, because for some observations, the sodium light can be filtered out. Sodium deposited in the upper atmosphere by micrometeorites naturally glows, so these wavelengths are already somewhat compromised for astronomy. Low-pressure sodium lights are in widespread use on the island of Hawaii where the Mauna Kea Observatory is located, and on La Palma in the Canary Islands, where the Roque de Los Muchachos Observatory is located. Rayleigh scattering of $589 \mathrm{~nm}$ light is much less than for light at bluer wavelengths.

High-pressure sodium lights emit light in a pressure broadened emission centered at $589 \mathrm{~nm}$. They offer better color rendition than low-pressure sodium lights, and are energy efficient. Because their emission is mostly yellow, orange and red light, Rayleigh scattering is less than for bluer light sources. 


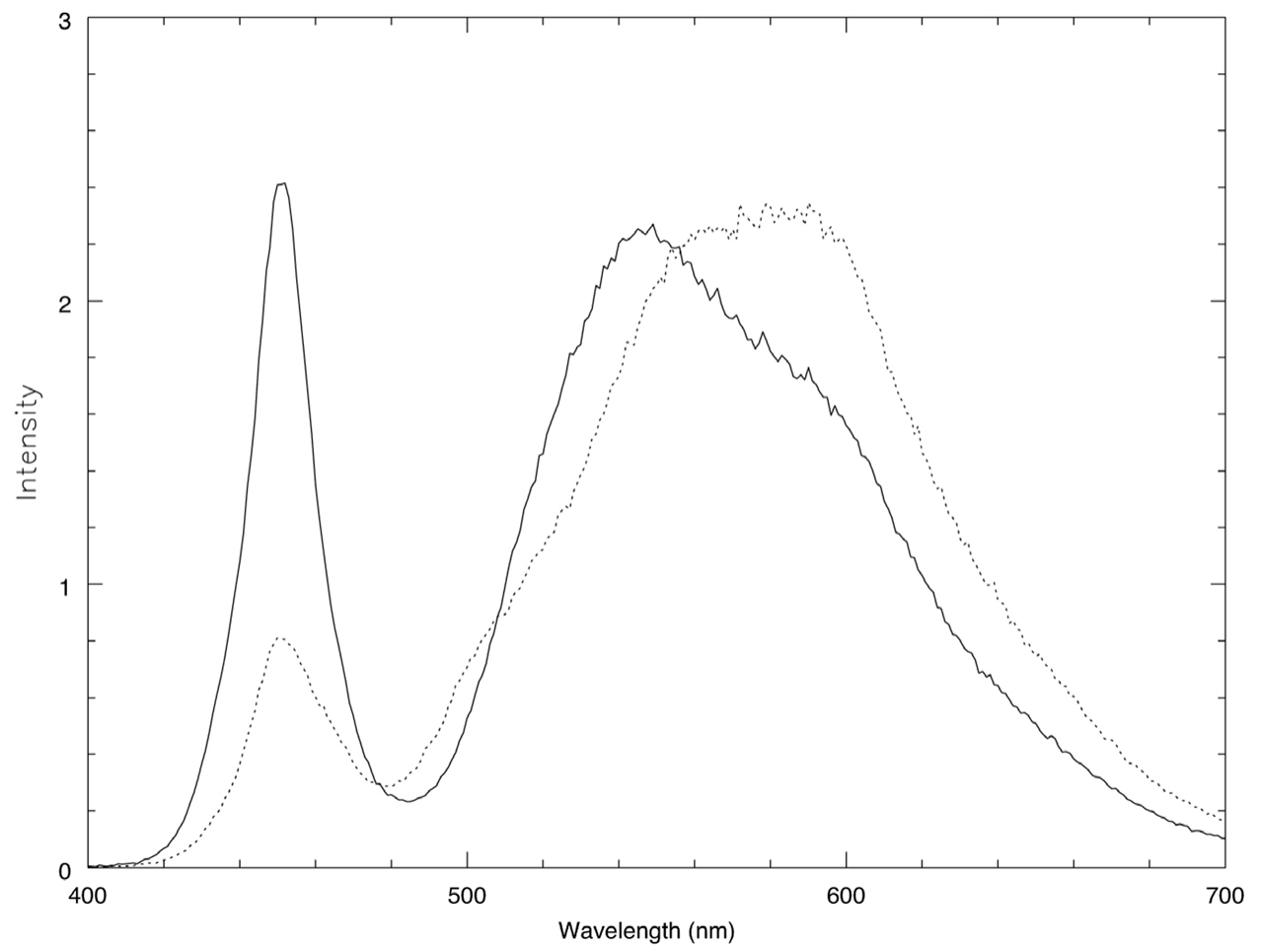

Figure 3. Spectra of white LEDs. The solid line shows the spectrum of a white LED with correlated color temperature (CCT) of 5,000K. The dotted line shows the spectrum of a white LED with CCT of $3,400 \mathrm{~K}$. The emission near $450 \mathrm{~nm}$ comes directy from the blue LED driving the light source. The emission from 500 to $700 \mathrm{~nm}$ comes from a phosphor that converts some of the blue light to redder colors. The 5,000K LED emits about three times more blue light than the $3,400 \mathrm{~K}$ LED.

White light sources, including mercury, fluorescent, induction, metal halide, and LEDs are much more damaging to astronomy, because of the bright emission lines that some of them have (e.g., mercury), and because of their blue light content that suffers from much higher Rayleigh scattering. Most observatories are at locations with clear air, so Rayleigh scattering is the dominant mechanism for producing artificial sky glow.

White LEDs present a new danger to astronomy and our ability to view the dark night sky. White LEDs have energy efficiencies that are approaching the energy efficiency of sodium lights. Very efficient light fixtures can be designed using LEDs, and LEDs are likely to require less maintenance than other types of light.

Most white LEDs are made from a blue LED and a phosphor that converts some of the blue light into green and red light. Typical spectra are shown in Figure 3. The blue emission peak near $450 \mathrm{~nm}$ comes from the blue LED emission, and occurs at a wavelength where the eye is not very sensitive, but astronomical instruments are very sensitive. Rayleigh scattering at $450 \mathrm{~nm}$ is almost 3 times more than at $589 \mathrm{~nm}$.

White lighting, and in particular white LED lighting, should be avoided near observatory sites. If white lights are used, low color temperatures $(3,000 \mathrm{~K}$ or lower) should be selected to reduce the damaging effects of the blue light that they emit. 


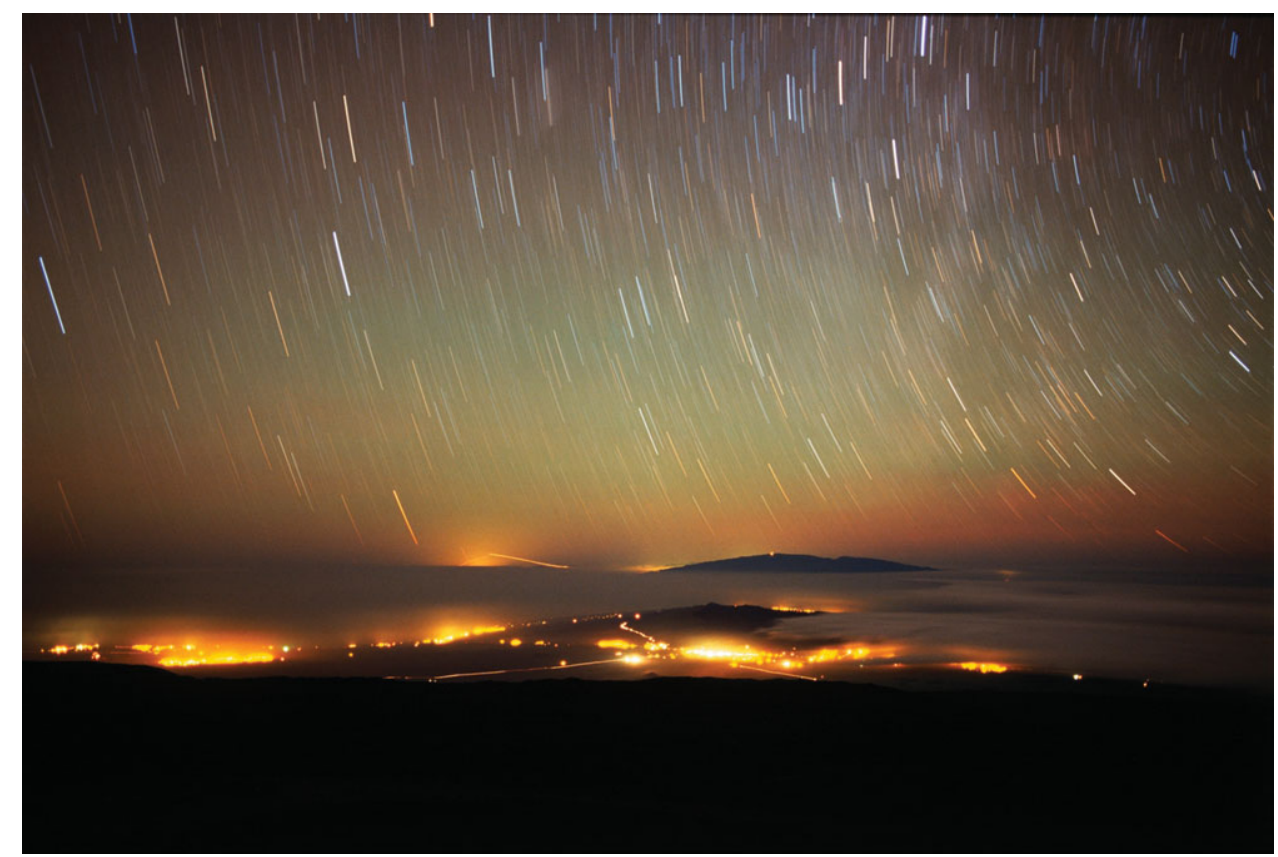

Figure 4. The view from Mauna Kea on a typical night. Direct emission from light sources that are not properly shielded is seen from the towns of Waikoloa (left) and Waimea (center). The majority of the lighting that is seen is low-pressure sodium lighting on the island of Hawaii. The eastern side of the Island (right side in this figure) is the wetter side of the island and is more frequently covered by low level clouds that reduce light pollution. The glow from Honolulu is near the horizon in the center-left, and a fainter glow from the island of Maui is to the right of Honolulu.

\section{Shielding of lighting}

Astronomers and the International Dark Sky Association have for many years been advocating careful shielding of lighting. Full shielding of lights prevents any light being emitted above the horizontal plane, directly into the atmosphere. If fully shielded lights are used, the only light that can cause sky glow comes from reflections from the ground.

Recently, some lighting manufacturers have suggested that by allowing a small amount of direct uplight, light spacing can be increased, and the total amount of light decreased. Luginbuhl, Walker, and Wainscoat (2009) have shown that light emitted upwards at angles close to the horizontal is extremely damaging to the night sky compared to light reflected from the ground. The reason is quite simple - Earth's atmosphere is relatively thin, so light that is reflected upwards from the ground has a reasonably high likelihood of leaving the atmosphere without scattering, whereas light that is emitted near the horizontal has a near $100 \%$ likelihood of scattering. This means that use of fully shielded light fixtures is in nearly all cases the best way to control sky glow. Of course, limiting the amount of light used to only what is needed is also important.

Figure 4 shows the view from the summit of Mauna Kea. It shows that local light sources dominate over distant sources, but also shows the enormous distance that light can travel through the atmosphere. Honolulu is approximately $300 \mathrm{~km}$ from Mauna Kea, but its glow in the sky is clearly visible. 


\section{Population growth and mining}

The areas around most of the world's great observatories are experiencing rapid population growth. Growth in associated lighting in each of these areas can be seen by examining nighttime images from the Defense Meteorological Satellite from 1992 to 2003. Growth in population and lighting threatens observatories in Hawaii, Chile, the Canary Islands, and Arizona. As population grows near these observatories, it will become increasingly important to retrofit existing light fixtures with better shielded (i.e., fully shielded) light fixtures. The northern area of Chile is in the midst of an extensive retrofit of lighting in which better shielded lights are replacing poorly shielded lights. Lights are slowly being retrofitted in Hawaii and in the Canary Islands.

Lights from 24-hour mining operations also present a threat to observatory sites in Chile and in Arizona.

\section{Summary}

For much of the world's population, the view of the night sky is now obscured by light pollution. Increasing public awareness, including efforts to save energy, and concerns about the environment and public health may help to reduce the growth of lighting in the future. More careful selection of light fixtures, and better regulation of the amount of light being used can partially restore the view of the night sky from urban locations.

Most of Earth's great observatory sites are threatened by light pollution. Continued strong efforts are necessary to protect them, including possible protection of these sites as World Heritage Sites (Marin 2010). As the population near these observatories grows, existing lights need to be retrofitted with fully shielded lights, and the amount of light used needs to be carefully regulated.

Increasing use of white lights at night presents a new threat to our view of the night sky. Whenever white lights are used, low color temperatures should be used to minimize the amount of blue light and the associated Rayleigh scattering.

\section{References}

Luginbuhl, C., Walker, C., \& Wainscoat, R. J. 2009, Physics Today, 441, 32

Marin, C. 2010, this volume p. 449.

Rich, C. \& Longcore, T. 2006, (eds.), Ecological Consequences of Artificial Night Lighting (Washington, Covelo and London: Island Press). 